and $\beta^{2}$-galactosidase activity. However, these responses were absent or significantly reduced in endothelial cells isolated from Nox 2 knockout mice. In conclusion, metabolic disorders in particular hyperglycaemia and insulin resistance play an important role in mediating Nox2 activation and oxidative stress in multiple organs in ageing. Nox2 is involved in normal ageing process-associated vascular inflammation and oxidative damage of endothelial dysfunction.

\section{MYOCARDIAL NOX2 ACTIVITY REGULATES ATRIAL FIBRILLATION SUSCEPTIBILITY}

${ }^{1}$ Alexandra Mighiu*, ${ }^{2}$ Alice Recalde, ${ }^{1}$ Klemen Ziberna, ${ }^{1}$ Jillian Simon, ${ }^{3}$ Gil Bub, ${ }^{4}$ Ajay Shah, ${ }^{1}$ Barbara Casadei. ${ }^{1}$ University of Oxford; ${ }^{2}$ Universite de Bordeaux; ${ }^{3}$ McGill University; ${ }^{4}$ King's College London

10.1136/heartjnl-2017-311726.153

Background Gp91-containing NADPH oxidases (NOX2) are a significant source of reactive oxygen species (ROS) in the human atrial myocardium. An increase in NOX2 activity accompanies atrial fibrillation (AF) induction and electrical remodelling in animal models and predicts incident $\mathrm{AF}$ in humans; however, whether an increase in atrial NOX2 activity is necessary to create a substrate for AF remains to be demonstrated. The purpose of this study is to determine whether an increase in NOX2-derived ROS directly contributes to the development of $\mathrm{AF}$ and to identify the molecular changes that occur downstream of NOX2 activation in atrial myocytes.

Methods and Results Mice with myocardial NOX2 overexpression (NOX2-Tg) showed a 2-fold increase in NADPH-stimulated superoxide production (2-hydroxyethidium by HPLC) in both the left and right atria, without any other electrophysiological or structural abnormalities (assessed by surface ECG and echocardiography); however, AF susceptibility assessed in vivo by transesophageal pacing was significantly higher in NOX2-Tg mice compared with their wild-type (WT) littermate controls (AF probability: $16.9 \pm 1.8 \%$ vs. $10.2 \pm 1.6 \%$ respecctively, $\mathrm{n}=25-26 /$ genotype, $\mathrm{p}<0.01)$. Moreover, oral supplementation with atorvastatin $(30 \mathrm{mg} / \mathrm{kg} / \mathrm{day})$, an inhibitor of NOX2 activity, substantially reduced atrial NADPH-stimulated ROS and AF susceptibility in NOX2-Tg mice. Ex vivo highresolution optical mapping of di-4-ANEPPS-stained atrial preparations revealed no differences in the action potential duration of left and right atria of NOX2-Tg mice compared to controls, suggesting that electrical arrhythmogenic remodelling does not occur with NOX2 overexpression. However, abnormalities in intracellular $\mathrm{Ca}^{2+}$ handling have also been linked to AF; intriguingly, western blot analyses of atrial tissues revealed a $28 \%$ reduction $(n=11-23 /$ genotype, $p=0.0032$ with un-paired students t-test) in the phosphorylation status of the ryanodine receptor (RyR) at Ser2814 in the right, but not the left atria of NOX2-Tg mice. Our ongoing experiments are now aimed at determining whether NOX2-derived ROS alters diastolic calcium leak from the sarcoplasmic reticulum in isolated atrial myocytes due to altered RyR gating.

Conclusions Myocardial NOX2 overexpression and related increase in NADPH-stimulated ROS production are associated with increased AF susceptibility in vivo and reduced RyR phosphorylation at Ser2814. These findings suggest increased
NOX2 ROS may promote arrhythmogenesis by modulating atrial calcium handling.

\section{HIGHER IGM ANTI OXIDISED LDL ANTIBODIES POINT TO FAVOURABLE PLAQUE CHARACTERISTICS AS DETERMINED BY RADIO FREQUENCY INTRAVASCULAR ULTRASOUND (RF-IVUS) AND NEAR INFRARED SPECTROSCOPY (NIRS) IN THE INTEGRATED IMAGING AND BIOMARKER STUDY 3 (IBIS-3)}

${ }^{1}$ Victor van den Berg, ${ }^{2}$ Dorian Haskard, ${ }^{1}$ Isabella Kardys, ${ }^{2}$ Mikhail Anan, ${ }^{1}$ Martijn Akkerhuis, ${ }^{1}$ Robert-Jan van Geuns, ${ }^{1}$ Peter de Jaegere, ${ }^{1}$ Nicolas van Mieghem, ${ }^{1}$ Evelyn Regar, ${ }^{1}$ Jurgen Ligthart, ${ }^{3}$ Victor Umans, ${ }^{2}$ Patrick Serruys, ${ }^{1}$ Eric Boersma, ${ }^{2}$ Ramzi Khamis*. ${ }^{1}$ Erasmus $M C_{i}^{2}$ Imperial College; ${ }^{3}$ Northwest Clinics

10.1136/heartjnl-2017-311726.154

Background Malondialdehyde (MDA)-LDL is one of the main oxidation products involved in atherosclerosis pathogenesis. IgM antibodies against MDA-LDL have been generally regarded as protecting from atherosclerosis. In contrast, the role of $\mathrm{IgG}$ antibodies is less clear. How antibody levels relate to coronary atherosclerosis plaque characteristics still needs to be determined.

Hypothesis We tested the hypothesis that low IgM anti-MDA antibody levels in serum are associated with plaques with larger lipid-laden necrotic cores.

Method IBIS-3 was a prospective cohort study that was designed to determine the ability of rosuvastatin to decrease necrotic core volume in coronary atherosclerosis. Patients undergoing coronary artery grafting or percutaneous intervention for (un)stable angina pectoris or myocardial infarction were eligible. After the standard procedure, radiofrequency intravascular ultrasound (RF-IVUS) and near-infrared spectroscopy (NIRS) measurements were performed in a non-culprit coronary artery with a diameter stenosis $<50 \%$. After a median of 386 days of high dose rosuvastatin treatment, imaging was repeated on the same segment. Antibodies against MDA-LDL, as well as total serum $\operatorname{IgM}$ and $\operatorname{IgG}$, were measured by ELISA. Associations between antibodies and the RF-IVUS and NIRS parameters were assessed using linear regression models with quartiles as independent variables and with further adjustments for age, sex, diabetes, smoking, LDL and HDL cholesterol and previous use of statins.

Results A total of 143 patients of the included patients had both blood samples and RF-IVUS measurements available, and NIRS was also performed on 90 of these. Mean age was 59.6 (9.0) years $(84.6 \%$ male) and $94.4 \%$ were already on a regular statin before inclusion. At baseline, IgM anti MDA-LDL antibody levels had a strong independent inverse relationship with lesional necrotic core volume $(\mathrm{p}=0.027)$ and percentage $(p=0.011)$ as well as with lipid core burden index (LCBI) $(p=0.024)$ in the worst $4 \mathrm{~mm}$ segment. This relationship was partially dependent on total serum IgM, as higher IgM levels also reflected a favourable necrotic core percentage on RFIVUS. There was no correlation between any imaging parameter and $\operatorname{IgG}$ antibodies, total serum IgG, HDL-cholesterol or LDL-cholesterol. Although total atheroma burden increased over one year, there was no change in necrotic core volume. 
Conclusion The data show that higher levels of IgM antibodies, including IgM anti-MDA, are associated with a decreased coronary necrotic core volume and lipid core burden, whereas total serum IgG and IgG anti-MDA LDL antibodies were not related to the measured plaque characteristics. The ability of IgM antibodies but not HDL- or LDL-cholesterol levels to indicate these important plaque characteristics is consistent with a proposed mechanistic role.

\section{INDUCERS OF PULMONARY ARTERIAL HYPERTENSION UPREGULATE THE EXPRESSION OF PLASMA MEMBRANE CALCIUM ATPASE 1 IN PULMONARY ARTERY SMOOTH MUSCLE CELLS}

${ }^{1}$ Jude C Ihugba*, ${ }^{1}$ Sathishkumar Kurusamy, ${ }^{2}$ Nadine Arnold, ${ }^{1}$ Priscille PC Polla, ${ }^{3}$ James Cotton, ${ }^{4,5,6}$ Pablo Gomez-del Arco, ${ }^{4,5}$ Juan Miguel Redondo, ${ }^{2}$ Allan Lawrie, ${ }^{1}$ Angel Luis Armesilla. 'Cardiovascular Molecular Pharmacology Laboratory, School of Pharmacy, Research Institute in Healthcare Science, Faculty of Science and Engineering, University of Wolverhampton, Wolverhampton, UK; ${ }^{2}$ Pulmonary Vascular Research Group, Infection, Immunity and Cardiovascular Disease, University of Sheffield, Sheffield, UK; ${ }^{3}$ Department of Cardiology, Heart and Lung Centre, New Cross Hospital, Wolverhampton, UK; ${ }^{4}$ Gene Regulation in Cardiovascular Remodelling and Inflammation Group, Centro Nacional de Investigaciones Cardiovasculares, Madrid, Spain; ${ }^{5} \mathrm{CIBERCV} ;{ }^{6}$ Department of Molecular Biology, Universidad Autonoma de Madrid, CBM-SO, Madrid, Spain

\subsection{6/heartjnl-2017-311726.155}

Pulmonary arterial hypertension (PAH) is a chronic and lifethreatening disease with high morbidity and mortality in adult and paediatric patients. PAH is characterised by a progressive narrowing and occlusion of small pulmonary arteries leading to increased pulmonary resistance, right ventricular hypertrophy, and, finally, right ventricular failure.

A large body of data has shown that proliferation and migration of pulmonary arterial smooth muscle cells (PASMCs) represent key events in the vascular remodelling of pulmonary arteries that occurs during PAH. Levels of cytoplasmic calcium are an important determinant of PASMC proliferation and migration, and failure in maintaining appropriate levels of intracellular calcium are associated with PAH. The plasma membrane calcium ATPase (PMCA) proteins extrude calcium from the cytosol to the extracellular medium, and in doing so, play a critical role in the modulation of intracellular calcium levels. In this work, we have investigated whether inducers of $\mathrm{PAH}$ trigger any changes in the expression of PMCA proteins in PASMCs.

Analysis of RNA expression levels for PMCA genes has revealed that treatment of PASMCs with PDGF results in a significant increase in the level of the RNA encoding for the protein PMCA1. Interestingly, PMCA1 RNA levels were also elevated in lungs of rats with monocrotaline-induced PAH. No changes were observed in the RNA levels for PMCA4, the other major PMCA isoform expressed in PASMCs. Although previous studies on the regulation of PMCA1 gene expression have identified functional binding sites for the transcription factors NFAT in the PMCA1 promoter region, we show here that PDGF-mediated upregulation of PMCA1 transcriptional expression is independent of activation of the calcineurin/ NFAT signalling pathway.

Our results suggest the involvement of PMCA1 in PASMC deregulation during $\mathrm{PAH}$, although determination of the link between increased expression of PMCA1 and $\mathrm{PAH}$ requires further investigation.

\section{MYELOID EXPRESSION OF TRIB1 REGULATES THE POLARISATION STATE OF TISSUE RESIDENT MACROPHAGES THAT HAS CONSEQUENCES ON PLASMA LIPID AND METABOLIC HOMEOSTASIS}

${ }^{1}$ Jessica M Johnston*, 'Adrienn Angyal, ${ }^{1}$ Eva Hadadi, ${ }^{2}$ Stephen E Hamby, ${ }^{3}$ Robert Bauer, ${ }^{1}$ Zabran Ilyas, ${ }^{1}$ Daniel Szili, ${ }^{1}$ Markus Ariaans, ${ }^{1}$ Heather L Wilson, ${ }^{4}$ Ronald M Krauss, ${ }^{5}$ Daniel J Rader, ${ }^{2}$ Alison H Goodall, 'Sheila E Francis, ${ }^{1}$ Endre Kiss-Toth. ${ }^{1}$ University of Sheffield; ${ }^{2}$ University of Leicester; ${ }^{3}$ Columbia University; ${ }^{4}$ Children's Hospital Oakland Research Institute; ${ }^{5}$ University of Pennsylvania

\subsection{6/heartjnl-2017-311726.156}

Introduction Genome wide association studies have identified Tribbles-1 (TRIB1) to be significantly associated with all major plasma lipid traits and as a risk factor for ischaemic heart disease and myocardial infarction. Studies in mice using Trib1 full body $\mathrm{KO}$ and liver-specific over-expression and $\mathrm{KO}$ models have shown that hepatic expression of TRIB1 reduces circulating lipids. Additionally, Trib1 has been implicated as a regulator of alternatively activated macrophages. However the potential interplay between hepatocytes, macrophages and Trib1 remain unexplored.

This study aimed to assess whether myeloid Trib1 regulates tissue macrophage polarisation and investigate its consequences on plasma lipid homeostasis.

Methods We developed myeloid specific Trib1 conditional knockout (Trib1 fl/fl x Lyz2Cre; Trib1 ${ }^{\mathrm{KO}}$ ) and over-expressor mice (ROSA26Trib1.Tg x Lyz2Cre; Trib1 ${ }^{\mathrm{Tg}}$ ), thereby deleting or over-expressing Trib1 in myeloid cells. Plasma lipid levels were directly measured by ion mobility. Macrophage phenotype was characterised in the liver (Kupffer cells, KCs), adipose (ATMs) and BMDMs by qPCR and semi-quantitative immunofluorescence analysis. Western blotting was used to assess regulators of macrophage polarisation. Furthermore, microarray analysis of human monocyte derived macrophages (MDMs) was employed to identify potential TRIB1-regulated cytokines.

Results Loss of myeloid Trib1 increased levels of plasma triglyceride, VLDL-C $(\mathrm{p}<0.05)$ and promoted pro-inflammatory polarisation in KCs $(\mathrm{p}<0.01)$, ATMs $(\mathrm{p}<0.01)$ and BMDMs $(\mathrm{p}<0.05)$, while Trib1 ${ }^{T g}$ mice revealed opposing changes in all parameters assessed. Western blotting showed TRIB1 modulates protein levels of C/EBP- $\beta^{2}$ and $-\beta^{2}(p<0.05)$, both key regulators of macrophage polarisation, via the control of COP1 activity and miR-155 expression. Microarray analysis of MDMs indicated TRIB1 may regulate production of a number of pro-inflammatory cytokines that are implicated in fatty liver disease and adipocyte lipolysis. Reduced expression of these was confirmed in in Trib1 $1^{T g}$ BMDMs $(p<0.05)$.

Conclusions Myeloid Trib1 is a potent regulator of lipid homeostasis, the loss of which promotes inflammation in metabolic tissues. Our observations uncover a novel mechanism of KC-hepatocyte cross talk mediated through Trib1.

\section{QUANTIFYING MANGANESE-CALCIUM INTERACTION FOR OPTIMAL CARDIAC MANGANESE ENHANCED MRI}

${ }^{1}$ Nur Hayati Jasmin*, ${ }^{2}$ Thomas Roberts, ${ }^{2}$ John Connell, ${ }^{3}$ Mark Lythgoe, ${ }^{3}$ Daniel Stuckey. ${ }^{1}$ Center for Advanced Biomedical Imaging; ${ }^{2}$ UCL Centre for Advanced Biomedical Imaging: ${ }^{3}$ UCL Centre for Advanced Biomedical Imaging (CABI)

10.1136/heartjnl-2017-311726.157 